\title{
Discovering the Pitfall of Using Horse Radish Peroxidase in Enzyme Linked Immunosorbent Assays for Detection of Pollen Specific IgE
}

\author{
Kenneth W Lee, $\mathrm{PhD}^{1 *}$ and Robert E Esch, $\mathrm{PhD}^{1,2}$ \\ ${ }^{1}$ Stallergenes Greer, 639 Nuway Circle, Lenoir, North Carolina, USA \\ ${ }^{2}$ School of Natural Sciences, Lenoir-Rhyne University, Hickory NC, USA
}

${ }^{*}$ Corresponding author: Kenneth W. Lee, PhD, Stallergenes Greer, 639 Nuway Circle, Lenoir, NC 28645, USA; Tel: 828 759-7345; Fax: 828 754-7306; E-mail: ken.lee@stallergenesgreer.com

Received: October 28, 2021; Accepted: November 08, 2021; Published: November 16, 2021

\begin{abstract}
Enzyme Linked Immunosorbent assays incorporate a functional enzyme conjugate in at least one procedural step; horseradish peroxidase (HRP) and alkaline phosphatase (AP) are the most commonly used. Recent evaluations in our laboratory yielded disparaging results for pollen specific IgE between assays that incorporate a biotinylated anti-IgE primary tracer reagent and either streptavidin-horseradish peroxidase (SA-HRP) or streptavidin-alkaline phosphatase (SA-AP) as the enzyme secondary conjugate. A screen of 1008 randomly selected samples submitted by veterinarians for routine allergy testing identified 128 samples that yielded a response consistent with an expected classical profile (CP) of positive responses when evaluated with an anti-IgE-biotin primary tracer followed by SA-HRP as secondary tracer, and negative responses when tested without anti-IgE-Biotin. An additional 96 reactive samples yielded a non-classical profile (NP) of equal magnitude with or without the anti-IgE-biotin conjugated tracer. Adsorption of IgE from sera pools prepared from CP samples and heat inactivation of IgE reactivity in these pools readily reduced the signal evident in untreated pools. Similar treatment of NP pools had minimal effect on the signal generated. Inhibition evaluations using unconjugated biotin or streptavidin indicates that neither is involved in the aberrant reaction. However, inhibition evaluations with unconjugated heat inactivated HRP reduces the reactivity in the NP pool and that the reactivity evident in the CP pool is unaffected by free HRP inhibition. These results are consistent with the hypothesis that antibodies specific for an epitope on glycoproteins present in the allergen extracts cross reacts with a component of HRP and binds the secondary tracer molecule without interfering with its enzymatic reactivity. Collectively, the results provide evidence that warrants avoidance of ELISAs that incorporate HRP as the enzyme containing tracer reagent but confirms the functional utility of ELISAs that incorporate alkaline phosphatase as the report enzyme, for assays that are intended for detection of allergen specific antibodies.
\end{abstract}

Keywords: Allergen specific IgE, ELISA, Pollen allergy, Horse radish peroxidase, Alkaline phosphatase

\section{Introduction}

Horseradish peroxidase (HRP) is one of the most widely used enzymes in analytical applications. HRP readily combines with hydrogen peroxide $\left(\mathrm{H}_{2} \mathrm{O}_{2}\right)$ and the resultant $\left[\mathrm{HRP}-\mathrm{H}_{2} \mathrm{O}_{2}\right]$ complex can oxidize a wide variety of hydrogen donors. Consequently, the enzyme is often used in biochemistry applications such as immunohistochemistry [1], western blots [2], and ELISA [3], where it is used to amplify a weak signal and increase detectability of a target molecule. In the diagnostic arena, HRP is widely used as an enzyme label in medical diagnostics and research applications. One such use is for identifying allergen specific IgE and multiple assays have been developed in both human and veterinary arenas [4-7].

It has been more than three decades since the first in vitro assay for detection of allergen specific IgE in dogs became commercially available and the functional characteristics of this assay were described [5]. The introduction of this enzyme-linked immunosorbent assay (ELISA) fostered a number of similar commercial assays, all of which rely upon the use of either polyclonal or monoclonal anti-IgE antibodies, or a recombinant human high-affinity IgE epsilon receptor fragment as primary tracer molecules [4-8]. Many of these assays incorporated HRP as the reporter enzyme while others used alkaline phosphatase conjugates. Comparative evaluations of these different tests demonstrated substantial variance of results for the various assays [914], but no efforts have been put forth to identify why such dramatic differences might exist. To address this issue more thoroughly, we opted to comparatively evaluate the responses of results evident in ELISA that incorporate either HRP or alkaline phosphatase (AP). We hypothesize that the differences in responses evident in the two assays resides in the binding differences of the reporter enzyme conjugates.

\section{Materials and Methods}

Sera

The serum samples used throughout were derived from dogs suspected of clinical allergy and had previously been submitted by veterinarians for evaluation using Stallergenes Greer macELISA for detection of allergen-specific IgE. The sole criterion for selecting 
individual samples for this study was the volume of sera remaining following the allergen testing exceeded $2.0 \mathrm{~mL}$; but hemolyzed or lipemic sera were excluded. Samples were stored frozen $\left(-20^{\circ} \mathrm{C}\right)$ for up to one year before being used in this study. A total of 1008 sera derived from individual dogs were each screened, in triplicate, on an allergen panel that contained extracts derived from ash, marsh elder, and ragweed pollens. All samples were screened using an HRP based ELISA, and each was evaluated with and with and without anti-IgEbiotin included in the assay. Following the initial evaluation, two separate sera pools were prepared based on the reactivity profiles evident when evaluated in the HRP ELISA. Subsequent evaluations of the sera pools were completed using both HRP and AP based ELISAs, and a portion of each pool was heated at $56^{\circ} \mathrm{C}$ to inactivate the functional binding of IgE in the assays.

\section{Buffers}

The buffers used throughout have been previously described, $[6,15,16]$ and included: a) well coating buffer: $0.05 \mathrm{M}$ sodium carbonate bicarbonate buffer, $\mathrm{pH} 9.6$; b) wash buffer: phosphate buffered saline (PBS), pH 7.4, containing $0.05 \%$ Tween 20 , and $0.05 \%$ sodium azide; c) serum and reagent diluent buffer: PBS, $\mathrm{pH} 7.4$, containing $1 \%$ fish gelatin, $0.05 \%$ Tween 20 and $0.05 \%$ sodium azide.

\section{Preparation of Coated Wells}

Immulon $4 \mathrm{HBH}$ flat bottom strip assemblies (Thermo Electron Corporation, Waltham, MA) were used throughout and served as the solid phase for all ELISA evaluations. The twelve well strips were individually coated with the specified allergen extracts following a previously defined procedure [6]. Briefly, the individual extracts were diluted in bicarbonate buffer ( $\mathrm{pH} 9.6)$ and $100 \mu \mathrm{L}$ was added to each assigned well. Following overnight incubation at $4-8^{\circ} \mathrm{C}$, the wells were washed with PBS, blocked with $1 \%$ monoethanolamine (pH 7.5) then air dried and stored at $4-8^{\circ} \mathrm{C}$ in resealable plastic bags until used. The allergen panel used for screening samples consisted of ash, marsh elder, and ragweed allergens. To compare HRP and AP based ELISA a panel that included 10 grasses, 10 weeds, and 4 trees was used.

\section{IgE Detection Reagents}

Monoclonal anti-IgE antibodies were biotinylated using EZLink Sulfo-NHS-LC-Biotin ester (Thermo Scientific, Waltham, MA, USA) following the manufacturer's recommended procedure. Briefly, individual monoclonal anti-IgE solutions were dialyzed against 50 $\mathrm{mM}$ carbonate buffer $\mathrm{pH}$ 8.5. A $6 \mathrm{mM}$ solution of biotin ester in bicarbonate buffer was added to each monoclonal solution to yield a molar ratio mixture of 12:1 (biotin: monoclonal). Following incubation at room temperature for 2 hours with constant agitation, excess biotin ester was removed by dialysis against Tris-Buffered Saline. The protein recovered from each reaction mixture was estimated by determining the optical density at $278 \mathrm{~nm}$, assuming an extinction coefficient of 1.4 for each monoclonal IgG. A sufficient volume of glycerol was added to each monoclonal-biotin conjugate to yield a $50 \%$ solution before storage at $-20^{\circ} \mathrm{C}$. The IgE specificity of the monoclonal anti-IgE was confirmed by documenting the heat lability $\left(56^{\circ} \mathrm{C}\right)$ of reactive serum components. A mixture of three monoclonal anti-canine IgE antibody preparations was optimized for use in the IgE specific ELISAs.
The substrate reagent used for the SA-HRP conjugate, $o$-phenylenediamine dihydrochloride (OPD), was purchased from Sigma-Aldrich (St. Louis, MO, USA). The SA-AP substrate reagent, $p$-nitrophenylphosphate $(p \mathrm{NPP})$, was purchased from Moss Inc (Pasadena, MD, USA). Each enzyme conjugate was stored at $4-8{ }^{\circ} \mathrm{C}$ in its respective stabilizing buffer, which was also purchased (SigmaAldrich, St. Louis, MO, USA).

\section{Sample Evaluations - ELISA}

The basic operational characteristics and procedures for the ELISAs have been previously described $[5,6]$. Briefly, serum samples were diluted 1:6 in diluent buffer. One hundred microlitres of the diluted samples was added to coated microwells and incubated overnight $(14-16 \mathrm{~h})$ at $4-8^{\circ} \mathrm{C}$. The wells were washed twice with PBS-T, and $100 \mu \mathrm{L}$ of biotinylated monoclonal anti-IgE-biotin mixture in diluent buffer was added to each well; when evaluated without antiIgE-biotin $100 \mu \mathrm{L}$ of diluent buffer was substituted for the biotinylated monoclonal anti-IgE-biotin mixture. After $2 \mathrm{~h}$ incubation at room temperature $\left(22^{\circ} \mathrm{C}\right)$, the wells were washed thrice with PBS-T, and 100 $\mu \mathrm{L}$ of streptavidin-enzyme conjugate in diluent buffer was added to each well before incubation for $1 \mathrm{~h}$ at room temperature. Following a final washing (four cycles with PBS-T), $100 \mu \mathrm{L}$ of appropriate substrate was added to each well. Following a $1 \mathrm{~h}$ incubation period, the HRP reactivity was stopped by adding $50 \mu \mathrm{L}$ of $2 \mathrm{M} \mathrm{H}_{2} \mathrm{SO}_{4}$ to each well while the AP reactivity was stopped by adding $50 \mu \mathrm{L}$ of $20 \mathrm{mM}$ cysteine to each well. Specific IgE reactivity to the allergens was then estimated by determining the absorbance of each well measured at $405 \mathrm{nM}$ for the AP ELISA and $492 \mathrm{~nm}$ for HRP ELISA using an automated plate reader. All results are expressed as ELISA Absorbance Units (EAU) which are background-corrected observed responses expressed as milli absorbance [6].

\section{Statistics}

Statistical analysis was performed with a commercial software package (PRISM v9, GraphPad; La Jolla, CA, USA); $P$-values $\leq 0.05$ were considered statistically significant. The Student's t-test was used to evaluate the significance of differences of observed responses.

\section{Results}

\section{Definition of Classical and Non-Classical Responses}

The first experiments undertaken evaluated the reactivity evident in serum samples that were evaluated on an allergen panel that included extracts of ash, marsh elder and ragweed; each sample was evaluated with and without an anti-IgE-biotin tracer conjugate. For those sera samples that were shown to possess allergen specific IgE for these allergen extracts when evaluated in an ELISA that incorporates HRP as the reporter enzyme, two representative reactivity profiles became readily apparent. The first, which is representative of what might be expected when a serum sample is evaluated with or without anti-IgEbiotin included in the assay. The signal generated with the inclusion of anti-IgE-biotin was not evident when the assay was completed using diluent only in place of anti-IgE-biotin. We have identified this reaction profile as a classical profile (CP). The second response profile that is observed with some serum samples were characterized as a non- 
classical profile (NP). For these samples the magnitude of signal that was yielded, for all allergens in the screen, without including anti-IgEbiotin in the assay were of the same order of magnitude $(\mathrm{P}<0.001)$ as the signals generated when this reagent was included. Approximately $22 \%(224 / 1008)$ of all the samples screened were reactive to the pollen allergens (Table 1). Of these reactive samples, $57.2 \%$ (128/224) yielded a reactivity profile consisted with a classical response, and 42.8\% (96/224) responded in a non-classical manner. All subsequent evaluations presented in this document used pools of sera that were derived from these characterized samples. Only samples that exhibited a classical response were included in the CP sera pool; likewise, only samples that exhibited similar responses with and without anti-IgE-biotin in the evaluation were included in the NP sera pool.

\section{Allergen Reactivity Profile for Classical and Non-Classical Sera Pools}

To characterize the reactivity profile for the CP and NP sera pools for a panel of grass, weed, and tree pollen allergens, each pool was evaluated using an HRP based ELISA as well as an AP based ELISA; each pool was evaluated with and without anti-IgE-biotin in the assay. The results demonstrate that the CP pool does, in fact, react to all pollens tested (Table 2) in a manner consistent with the definition of a classical reactivity profile. Signals of substantial magnitude were evident with all grasses, weeds, and trees, but the signals yielded without including anti-IgE-biotin were dramatically different $(\mathrm{P}<0.001)$ and were indistinguishable from the background responses $(\mathrm{P}<0.001)$. On the other hand, the signals evident with the NP pool were not significantly different $(\mathrm{P}=0.264)$ when evaluated with or without anti-IgE-biotin (Table 2). The average signal evident with the NP pool when evaluated on grass pollen allergens in the absence of anti-IgE-biotin was $76.7 \%$ of the signal that was generated in the presence of anti-IgE-biotin and ranged between 50.0-90.3\% depending on the allergen tested. When evaluated in the absence of anti-IgE-biotin, the average signal evident with weed and tree pollen allergens was $93.3 \%$ (range $84.3-100 \%$ ) and $87.8 \%$ (range 60.0 $99.2 \%$ ), respectively, of the signal evident when evaluated with the biotinylated reagent.

\section{Heat inactivation of IgE reactivity in sera}

The effect of heating on the reactivity of the CP and NP sera pools to the various allergens demonstrated the that a significant portion $(>90 \%$; $\mathrm{P}<0.001)$ of the pollen reactivity evident in the CP pool (presumably IgE) was eliminated following heat treatment (Table 3 ).

A significant $(\mathrm{P}<0.001)$ portion of the pollen reactivity evident in the NP pool was also affected by heating; however, substantial reactivity (ca. 50\%) remained following heat treatment and the reactivity evident with and without anti-IgE-biotin were not different $(P=0.631)$. Similar profiles of reactivity were evident in the $\mathrm{CP}$ and NP pools when each pool was rendered deficient in IgE by immunoaffinity chromatography using solid phase bound anti-IgE (data not shown).

\section{Reactivity of Classical and Non-Classical Sera Pools When Reacted with Free HRP}

The blocking effects that free biotin, streptavidin, or HRP might have on the reactivity evident in the NP pool, but lacking in the $\mathrm{CP}$ pool, was evaluated by incubating each serum pool individually with an excess of each of these components. None of these treatments altered the reactivity profile of either of the CP or NP sera pools (data not shown). However, the results clearly demonstrated that evaluation of the NP sera pool with free HRP included in the assay, at varying concentrations, in place of the streptavidin-HRP conjugate, resulted in generation of a substantial signal that approximates one-half the magnitude of signal evident when the assay was completed including streptavidin-HRP (Figure 1).

No significant differences $(\mathrm{P}<0.001)$ were noted for the responses evident with or without anti-IgE-biotin regardless of the concentration of free HRP that was included in the assay. However, the signals evident at $31.3 \mathrm{nG} / \mathrm{mL}$ or less of free HRP were significantly different $(\mathrm{P}, 0.001)$ than the signals evident at concentrations of $250 \mathrm{ng} / \mathrm{ml}$ or greater which indicated a concentration dependent binding of the free HRP. A similar signal, presumably due to binding of the free HRP, was lacking in the CP sera pool. The addition of enzymatically inactive HRP had no dramatic effect on either the background responses (P, 0.001) or the signal generated with the $\mathrm{CP}$ pool when evaluated with or without anti-IgE-biotin (Figure 2). On the other hand, the magnitude of signal generated with the NP pool was dramatically diminished $(\mathrm{P}<0.001)$ when evaluated with or without anti-IgE-biotin.

\section{Reactivity of Classical Pool and Non-Classical Pool Using Alkaline phosphatase ELISA}

Finally, both CP and NP sera pools were evaluated using an ELISA that incorporates a streptavidin-alkaline phosphate conjugate in place of the streptavidin-HRP conjugate. The results (Table 4) demonstrated that a substantial signal was yielded with the CP sera pool when evaluated with anti-IgE-biotin and that the signal was lacking in the assay without anti-IgE-biotin $(\mathrm{P}<0.001)$. Similarly, no signal was evident $(\mathrm{P}<0.001)$ with the NP sera pool when evaluated without antiIgE-biotin. Yet, a substantial signal $(\mathrm{P}<0.001)$ indicative of allergen specific IgE was detected when using the alkaline phosphatase enzyme.

Table 1: Incidence of pollen reactivity that behave in expected classical manner and non-classical manner (N=1008).

\begin{tabular}{|l|c|c|c|}
\hline Samples & Number of Test Samples & \% of Screened Samples & \% of Test Reactive Samples \\
\hline Reactive to Test Antigens $^{\mathrm{a}}$ & 224 & 100 & 22.2 \\
\hline Reactive in Non-Classical Manner $^{\mathrm{b}}$ & 96 & 12.5 & 42.8 \\
\hline Reactive in Classical Manner $^{\mathrm{b}}$ & 128 & 57.2 & \\
\hline
\end{tabular}

${ }_{\text {a }}^{a}$ Tall Ragweed, Marsh Elder, and White Ash.

Samples reacting in non-classical manner are reactive with and without inclusion of the primary biotinylated tracer samples reacting in classical manner are reactive only when primary biotinylated tracer is included in the assay. 
Kenneth W Lee (2021) Discovering the Pitfall of Using Horse Radish Peroxidase in Enzyme Linked Immunosorbent Assays for Detection of Pollen Specific IgE

Table 2: Pollen reactivity of Classical and Non-Classical sera pools when evaluated with and without anti-IgE-biotin in an ELISA that incorporates HRP in the secondary tracer conjugate (mean $\pm \mathrm{SD}$ of triplicate evaluations).

\begin{tabular}{|c|c|c|c|c|}
\hline \multirow[t]{3}{*}{ Allergens } & \multirow{2}{*}{\multicolumn{2}{|c|}{ Classical Pool }} & & \\
\hline & & & \multicolumn{2}{|c|}{ Non Classical Pool } \\
\hline & Biotin & NO Biotin & Biotin & NO Biotin \\
\hline \multicolumn{5}{|l|}{ Grasses } \\
\hline Bermuda (Cynodon dactylon) & $3864 \pm 29$ & $6 \pm 2$ & $2266 \pm 25$ & $1871 \pm 54$ \\
\hline Brome (Bromus inermis) & $3844 \pm 50$ & $39 \pm 7$ & $926 \pm 25$ & $463 \pm 27$ \\
\hline Johnson (Sorghum halepense) & $3779 \pm 36$ & $0 \pm 1$ & $3246 \pm 40$ & $2931 \pm 102$ \\
\hline Kentucky Blue (Poa pratensis) & $3839 \pm 36$ & $46 \pm 10$ & $773 \pm 66$ & $458 \pm 69$ \\
\hline Meadow fescue (Festuca pratensis) & $3875 \pm 29$ & $123 \pm 37$ & $2200 \pm 85$ & $1818 \pm 70$ \\
\hline Orchard (Dactylis glomerata) & $3868 \pm 25$ & $99 \pm 32$ & $2305 \pm 53$ & $1853 \pm 94$ \\
\hline Perennial Rye (Lolium perenne) & $3854 \pm 32$ & $147 \pm 49$ & $1911 \pm 212$ & $1393 \pm 46$ \\
\hline Red Top (Agrostis alba) & $3855 \pm 62$ & $32 \pm 3$ & $2111 \pm 27$ & $1801 \pm 94$ \\
\hline Sweet Vernal (Anthoxanthum odoratum) & $3791 \pm 75$ & $101 \pm 25$ & $1585 \pm 123$ & $1317 \pm 131$ \\
\hline Timothy (Phleum pratense) & $3861 \pm 29$ & $34 \pm 10$ & $1708 \pm 38$ & $1373 \pm 89$ \\
\hline \multicolumn{5}{|l|}{ Trees } \\
\hline Birch (Betula nigra) & $2042 \pm 172$ & $33 \pm 56$ & $3031 \pm 78$ & $2937 \pm 51$ \\
\hline Box Elder (Acer negundo) & $3819 \pm 16$ & $0 \pm 6$ & $1170 \pm 37$ & $702 \pm 57$ \\
\hline Quaking Aspen (Populus tremuloides) & $3569 \pm 105$ & $0 \pm 19$ & $1683 \pm 44$ & $1670 \pm 70$ \\
\hline White Ash (Fraxinus Americana) & $2357 \pm 71$ & $155 \pm 15$ & $3229 \pm 53$ & $3076 \pm 41$ \\
\hline \multicolumn{5}{|l|}{ Weeds } \\
\hline Cocklebur (Xanthium strumarium) & $3493 \pm 49$ & $47 \pm 54$ & $3302 \pm 17$ & $2934 \pm 49$ \\
\hline English Plantain (Plantago lanceolata) & $3649 \pm 83$ & $22 \pm 16$ & $1660 \pm 28$ & $1431 \pm 113$ \\
\hline Kochia (Bassia scoparia) & $3809 \pm 115$ & $0 \pm 3$ & $3328 \pm 44$ & $3215 \pm 69$ \\
\hline Lambs Quarter (Chenopodium album) & $3717 \pm 55$ & $0 \pm 10$ & $3381 \pm 23$ & $3218 \pm 53$ \\
\hline Marsh Elder (Cyclachaena xanthiifolia) & $3781 \pm 19$ & $46 \pm 5$ & $3539 \pm 26$ & $3546 \pm 35$ \\
\hline Marsh Elder (Iva annua) & $2633 \pm 123$ & $88 \pm 13$ & $3553 \pm 48$ & $3540 \pm 24$ \\
\hline Pigweed (Amaranthus palmeri) & $3819 \pm 50$ & $52 \pm 8$ & $3271 \pm 40$ & $3032 \pm 39$ \\
\hline Ragweed (Ambrosia trifida) & $3839 \pm 10$ & $68 \pm 18$ & $3474 \pm 18$ & $3418 \pm 40$ \\
\hline Sheep Sorrel (Rumex acetosella) & $3696 \pm 122$ & $68 \pm 73$ & $3117 \pm 3$ & $2902 \pm 148$ \\
\hline Yellow Dock (Rumex crispus) & $3663 \pm 100$ & $254 \pm 27$ & $2924 \pm 82$ & $2464 \pm 149$ \\
\hline
\end{tabular}

All results were expressed as ELISA Absorbance Units (EAU; mean \pm SD) which are background corrected observed responses (OD at $492 \mathrm{~nm})$ expressed as milli absorbance.

\section{Discussion}

We characterized two different patterns of reactivity among dog serum samples that yield a positive reaction in an ELISA that incorporates HRP as the reporter enzyme. The classical profile of reactivity was evident in samples that yield a positive response when evaluated in ELISA that include all assay components but exhibited no response when tested in the absence of the anti-IgE-biotin test reagent; slightly less than $60 \%$ of the reactive samples tested yield this characteristic response. The non-classical profile of reactivity was characteristic of samples that yielded a response in ELISA when evaluated without anti-IgE-biotin tracer reagent; more than $40 \%$ of the pollen reactive dog serum samples yielded a response with this characteristic. The magnitude of response evident in these samples approximated the magnitude of response evident when the sample was evaluated in an ELISA including anti-IgE-biotin reagent.
Pools of these respective sera yielded responses that were consistent with the responses evident with the individual samples that comprise the pools. When evaluated with and without the anti-IgE-biotin reagent the character of the $\mathrm{CP}$ and NP pools were evident with all pollen allergens tested, which encompass 10 grasses, 10 weeds, and 4 trees. However, when evaluated against mite allergen extracts the responses evident with both the $\mathrm{CP}$ and NP pools were indistinguishable from background responses when evaluated in the absence of the anti-IgE-biotin reagent (data not shown). Thus, this serum dependent response appears to be restricted to pollen extracts. Neither the functional removal of IgE from the sera pools by heat treatment nor the physical removal of IgE using immunoaffinity chromatography substantially altered the response profile yielded with the NP pool. However, the magnitude of responses that were evident in the NP pool following heat treatment were reduced by $40 \%$ to $50 \%$ of the signal evident in the unheated sample, indicating that a portion 
Kenneth W Lee (2021) Discovering the Pitfall of Using Horse Radish Peroxidase in Enzyme Linked Immunosorbent Assays for Detection of Pollen Specific IgE

Table 3: Pollen reactivity of Classical and Non-Classical sera pools following heat treatment $\left(56^{\circ} \mathrm{C}, 4 \mathrm{~h}\right)$ when evaluated with and without anti-IgE-biotin in an ELISA that incorporates HRP in the secondary tracer conjugate.

\begin{tabular}{|c|c|c|c|c|}
\hline \multirow[t]{3}{*}{ Allergens } & \multirow{2}{*}{\multicolumn{2}{|c|}{ Classical Pool }} & & \\
\hline & & & \multicolumn{2}{|c|}{ Non Classical Pool } \\
\hline & Biotin & NO Biotin & Biotin & NO Biotin \\
\hline \multicolumn{5}{|l|}{ Grasses } \\
\hline Bermuda (Cynodon dactylon) & $264 \pm 51$ & $17 \pm 11$ & $895 \pm 39$ & $869 \pm 42$ \\
\hline Brome (Bromus inermis) & $261 \pm 47$ & $71 \pm 11$ & $327 \pm 12$ & $333 \pm 71$ \\
\hline Johnson (Sorghum halepense) & $174 \pm 21$ & $0 \pm 55$ & $1702 \pm 56$ & $1693 \pm 48$ \\
\hline Kentucky Blue (Poa pratensis) & $246 \pm 7$ & $31 \pm 9$ & $258 \pm 73$ & $179 \pm 55$ \\
\hline Meadow fescue (Festuca pratensis) & $282 \pm 6$ & $53 \pm 4$ & $943 \pm 8$ & $708 \pm 8$ \\
\hline Orchard (Dactylis glomerata) & $241 \pm 3$ & $92 \pm 15$ & $1001 \pm 4$ & $806 \pm 31$ \\
\hline Perennial Rye (Lolium perenne) & $282 \pm 8$ & $74 \pm 18$ & $686 \pm 39$ & $478 \pm 29$ \\
\hline Red Top (Agrostis alba) & $236 \pm 26$ & $30 \pm 6$ & $924 \pm 75$ & $927 \pm 82$ \\
\hline Sweet Vernal (Anthoxanthum odoratum) & $272 \pm 25$ & $295 \pm 21$ & $717 \pm 62$ & $706 \pm 47$ \\
\hline Timothy (Phleum pratense) & $200 \pm 6$ & $24 \pm 4$ & $665 \pm 13$ & $435 \pm 7$ \\
\hline \multicolumn{5}{|l|}{ Trees } \\
\hline Birch (Betula nigra) & $139 \pm 43$ & $37 \pm 6$ & $1879 \pm 37$ & $1818 \pm 53$ \\
\hline Box Elder (Acer negundo) & $190 \pm 43$ & $7 \pm 10$ & $527 \pm 45$ & $374 \pm 28$ \\
\hline Quaking Aspen (Populus tremuloides) & $103 \pm 5$ & $1 \pm 41$ & $687 \pm 45$ & $747 \pm 39$ \\
\hline White Ash (Fraxinus Americana) & $246 \pm 4$ & $160 \pm 11$ & $2115 \pm 26$ & $2051 \pm 9$ \\
\hline \multicolumn{5}{|l|}{ Weeds } \\
\hline Cocklebur (Xanthium strumarium) & $167 \pm 22$ & $52 \pm 11$ & $1560 \pm 130$ & $1217 \pm 169$ \\
\hline English Plantain (Plantago lanceolata) & $198 \pm 22$ & $9 \pm 7$ & $631 \pm 16$ & $431 \pm 17$ \\
\hline Kochia (Bassia scoparia) & $219 \pm 8$ & $28 \pm 1$ & $1967 \pm 89$ & $2016 \pm 33$ \\
\hline Lambs Quarter (Chenopodium album) & $136 \pm 25$ & $0 \pm 9$ & $2302 \pm 57$ & $1977 \pm 29$ \\
\hline Marsh Elder (Cyclachaena xanthiifolia) & $162 \pm 1$ & $24 \pm 11$ & $2730 \pm 198$ & $2867 \pm 50$ \\
\hline Marsh Elder (Iva annua) & $157 \pm 13$ & $45 \pm 6$ & $2872 \pm 64$ & $2969 \pm 19$ \\
\hline Pigweed (Amaranthus palmeri) & $258 \pm 40$ & $56 \pm 6$ & $1917 \pm 15$ & $1451 \pm 41$ \\
\hline Ragweed (Ambrosia trifida) & $189 \pm 7$ & $55 \pm 1$ & $2518 \pm 81$ & $2556 \pm 71$ \\
\hline Sheep Sorrel (Rumex acetosella) & $244 \pm 28$ & $25 \pm 2$ & $1757 \pm 51$ & $1449 \pm 68$ \\
\hline Yellow Dock (Rumex crispus) & $396 \pm 20$ & $181 \pm 6$ & $1380 \pm 80$ & $1134 \pm 51$ \\
\hline
\end{tabular}

of the response evident in the NP pool was likely the result of allergen specific IgE. Greater than $90 \%$ of the allergen specific IgE present in the CP pool was inactivated by heating and substantiates that the reactivity remaining in the NP pool following heat treatment was due to a serum component that is not IgE with specificity toward the pollen allergens. This conclusion is supported by the results observed following removal of IgE from the sera pools.

To define a plausible explanation for the results observed with this non-classical response we attempted to block the response using various unconjugated assay components that might be interacting with the responsible serum component, and in so doing allowed for the binding of the HRP-streptavidin enzyme conjugate component of the assay. Addition or substitution of any of the assay components with the unconjugated reactive motif (biotin, streptavidin, or HRP) did not alter the reactivity profile evident with either the CP of NP pools. However, substitution of Streptavidin-HRP with unconjugated, but enzymatically active, HRP yielded results consistent with the NP reactivity profile but had no effect on the CP pool. Such results were consistent with the hypothesis that a serum component evident in the NP pool was facilitating HRP (either conjugated or unconjugated) binding during the streptavidin-HRP incubation stage of the assay. To address this hypothesis, heat treated $\left(90{ }^{\circ} \mathrm{C}, 4\right.$ hrs.) HRP was simultaneously added, along with the streptavidinHRP, at the appropriate stage of the assay. The results demonstrated that functionally inactive HRP does, in fact, substantially reduced the signal that was generated with the NP pool but did not alter the signal evident with the CP pool. These results, combined with the results yielded using a comparable ELISA that uses alkaline phosphate enzyme conjugate, provide conformation that a serum component in the NP pool was effectively binding the HRP component of the streptavidin-HRP reagent. The interaction between the streptavidin and the serum dependent component does not modify the enzymatic functionality of HRP. 


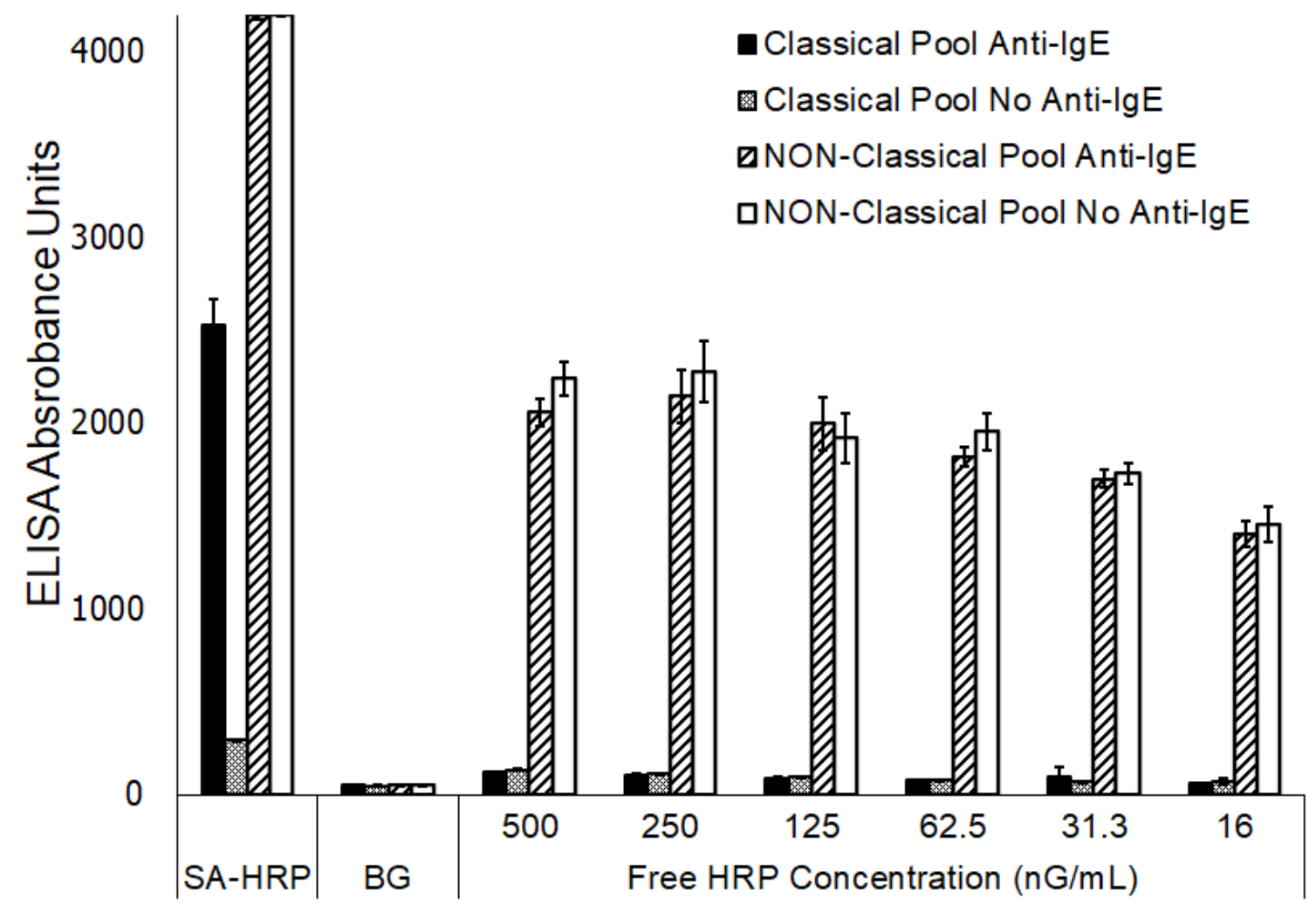

Figure 1: ELISA reactivity of Classical and Non-Classical sera pools to ragweed (mean \pm SD of triplicate evaluations) following substitution of Streptavidin-HRP with varying concentrations of unconjugated horseradish peroxidase.

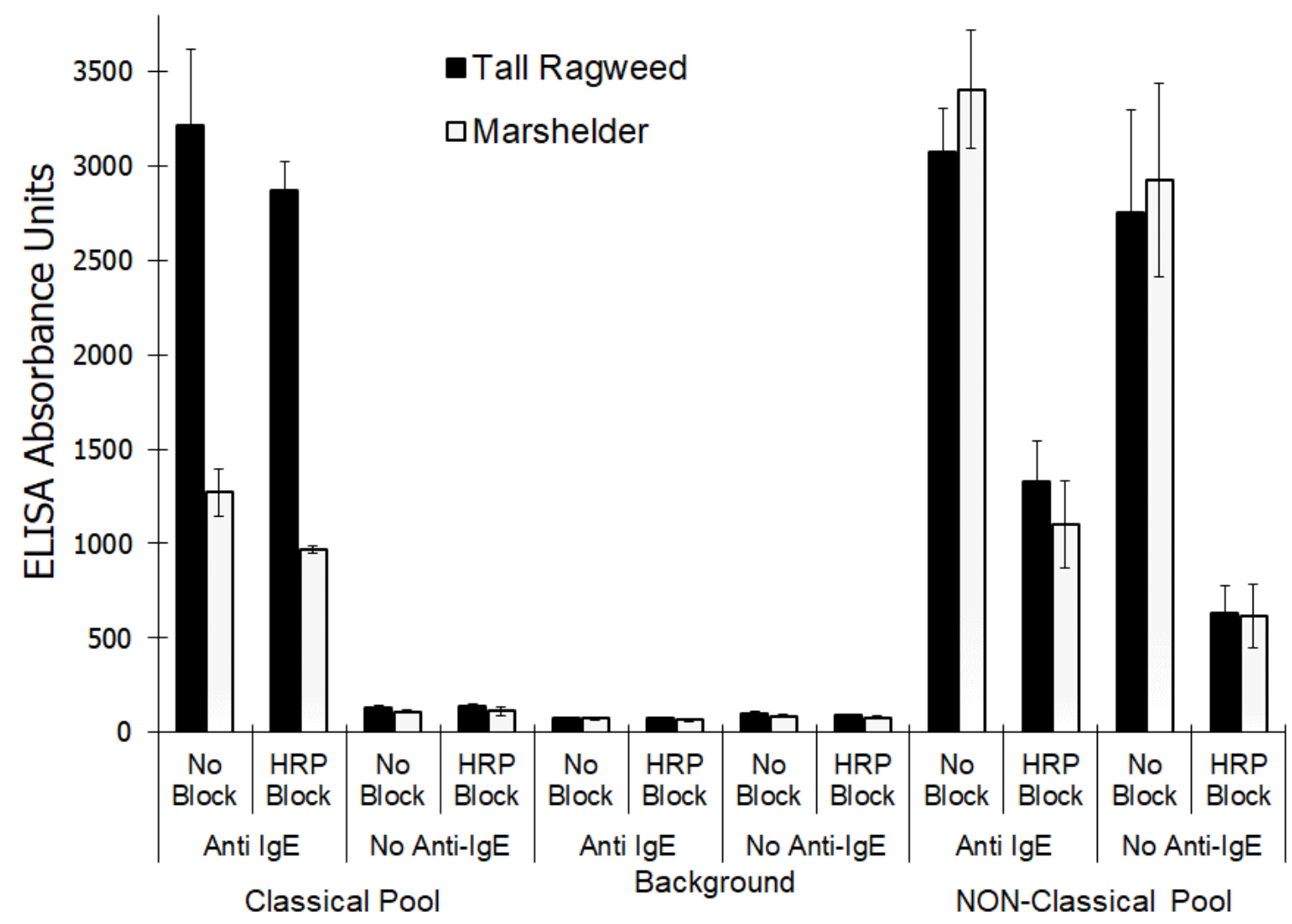

Figure 2: Effect of adding inactivated horseradish peroxidase on the reactivity of sera pools that exhibit Classical and Non-Classical reactivity profiles when evaluated using an ELISA (mean \pm $\mathrm{SD}$ of triplicate evaluations) that incorporates horse radish peroxidase enzyme conjugate. 
Kenneth W Lee (2021) Discovering the Pitfall of Using Horse Radish Peroxidase in Enzyme Linked Immunosorbent Assays for Detection of Pollen Specific IgE

Table 4: Pollen reactivity of Classical and Non-Classical sera pools when evaluated with and without anti-IgE-biotin in an ELISA that incorporates alkaline phosphatase in the secondary.

\begin{tabular}{|c|c|c|c|c|}
\hline \multirow[t]{3}{*}{ Allergens } & \multirow{2}{*}{\multicolumn{2}{|c|}{ Classical Pool }} & & \\
\hline & & & \multicolumn{2}{|c|}{ Non Classical Pool } \\
\hline & Biotin & NO Biotin & Biotin & NO Biotin \\
\hline \multicolumn{5}{|l|}{ Grasses } \\
\hline Bermuda (Cynodon dactylon) & $3835 \pm 58$ & $6 \pm 2$ & $835 \pm 10$ & $3 \pm 9$ \\
\hline Brome (Bromus inermis) & $3868 \pm 23$ & $39 \pm 7$ & $304 \pm 10$ & $22 \pm 3$ \\
\hline Johnson (Sorghum halepense) & $3789 \pm 28$ & $0 \pm 1$ & $1190 \pm 16$ & $0 \pm 10$ \\
\hline Kentucky Blue (Poa pratensis) & $3827 \pm 53$ & $46 \pm 10$ & $236 \pm 26$ & $39 \pm 88$ \\
\hline Meadow fescue (Festuca pratensis) & $3858 \pm 33$ & $123 \pm 37$ & $815 \pm 34$ & $24 \pm 9$ \\
\hline Orchard (Dactylis glomerata) & $3871 \pm 19$ & $99 \pm 32$ & $857 \pm 21$ & $39 \pm 7$ \\
\hline Perennial Rye(Lolium perenne) & $3845 \pm 51$ & $147 \pm 49$ & $699 \pm 85$ & $0 \pm 3$ \\
\hline Red Top (Agrostis alba) & $3821 \pm 23$ & $32 \pm 3$ & $782 \pm 11$ & $69 \pm 14$ \\
\hline Sweet Vernal (Anthoxanthum odoratum) & $3783 \pm 40$ & $101 \pm 25$ & $556 \pm 49$ & $57 \pm 9$ \\
\hline Timothy (Phleum pratense) & $3837 \pm 43$ & $34 \pm 10$ & $620 \pm 15$ & $25 \pm 12$ \\
\hline \multicolumn{5}{|l|}{ Trees } \\
\hline Birch (Betula nigra) & $2042 \pm 172$ & $33 \pm 56$ & $1099 \pm 31$ & $0 \pm 8$ \\
\hline Box Elder (Acer negundo) & $3819 \pm 16$ & $0 \pm 6$ & $369 \pm 15$ & $0 \pm 1$ \\
\hline Quaking Aspen (Populus tremuloides) & $3569 \pm 105$ & $1 \pm 19$ & $560 \pm 17$ & $0 \pm 4$ \\
\hline White Ash (Fraxinus Americana) & $2357 \pm 71$ & $155 \pm 15$ & $1162 \pm 21$ & $0 \pm 5$ \\
\hline \multicolumn{5}{|l|}{ Weeds } \\
\hline Cocklebur (Xanthium strumarium) & $3493 \pm 49$ & $47 \pm 54$ & $1252 \pm 7$ & $12 \pm 2$ \\
\hline English Plantain (Plantago lanceolata) & $3678 \pm 65$ & $22 \pm 16$ & $493 \pm 11$ & $0 \pm 7$ \\
\hline Kochia (Bassia scoparia) & $3794 \pm 71$ & $0 \pm 3$ & $1257 \pm 17$ & $40 \pm 1$ \\
\hline Lambs Quarter (Chenopodium album) & $3717 \pm 55$ & $0 \pm 10$ & $1218 \pm 9$ & $0 \pm 5$ \\
\hline Marsh Elder (Cyclachaena xanthiifolia) & $3780 \pm 19$ & $46 \pm 5$ & $1290 \pm 10$ & $0 \pm 5$ \\
\hline Marsh Elder (Iva annua) & $2633 \pm 123$ & $88 \pm 13$ & $1356 \pm 19$ & $14 \pm 1$ \\
\hline Pigweed (Amaranthus palmeri) & $3814 \pm 49$ & $52 \pm 8$ & $1228 \pm 16$ & $11 \pm 14$ \\
\hline Ragweed (Ambrosia trifida) & $3754 \pm 48$ & $68 \pm 18$ & $1299 \pm 7$ & $0 \pm 21$ \\
\hline Sheep Sorrel (Rumex acetosella) & $3751 \pm 68$ & $68 \pm 73$ & $1131 \pm 10$ & $0 \pm 4$ \\
\hline Yellow Dock (Rumex crispus) & $3713 \pm 57$ & $287 \pm 57$ & $1027 \pm 33$ & $0 \pm 2$ \\
\hline
\end{tabular}

All results were expressed as ELISA Absorbance Units (EAU) which are background corrected observed responses (OD at $405 \mathrm{~nm}$ ) expressed as milli absorbance.

The most obvious serum component that might allow for binding of HRP in an allergen specific ELISA without interaction of streptavidin-HRP conjugate with the anti-IgE biotinylated reagent is actually an allergen specific antibody. We propose that epitopes present on the pollen allergens that are cross-reactive with epitopes on HRP will specifically bind the various classes of antibodies, especially IgG, present in serum. During the subsequent incubation period with streptavidin-HRP the specific allergen bound antibody will also specifically bind to the cross-reactive epitope on the HRP molecule that is conjugated to the streptavidin but without interfering with the enzymatic function of HRP. With addition of substrate, a specific but non-related signal will result. Support for this hypothesis is the observation that antibodies specific for cross reactive carbohydrates (CCD) have been defined in a number of mammals including humans, dogs, and cats where the prevalence of anti-CCD IgE has been estimated to range from $20 \%$ to $70 \%$ [17-22]. Reaction with these molecules results in a false positive interpretation for many allergen extracts when evaluated using in vitro assays intended for detection of allergen specific IgE $[23,24]$. The relevant structure of the epitopes responsible for these false positive reactions has been characterized as a 1,3-fucose linked to the amide nitrogen of an asparagine residue of the protein $[25,26]$. Such carbohydrate containing structures have also been identified as specific carbohydrate epitopes of HRP. Furthermore, these specific N-glycans are widely distributed among pollens and invertebrate animals but are lacking in mammalian proteins $[25,26]$ where they can be strongly antigenic. This being the case, we propose that epitopes of this sort that are present on the pollen allergens will specifically bind the various classes of antibodies present in sera which then concomitantly binds the HRP conjugate.

Collectively, the results presented herein document substantial differences in responses that were yielded in an ELISA that incorporated an HRP enzyme conjugate and one that incorporated an AP enzyme conjugate. The results provide supportive information 
that warrants avoidance of ELISAs that incorporate HRP as the enzyme containing tracer reagent that are intended for detection of pollen specific antibodies. Also included are results that support the functional utility of ELISAs that incorporate alkaline phosphatase as the report enzyme.

\section{Acknowledgments}

Funding for this study was provided by Stallergenes Greer. At the time of the study all authors were employees of Stallergenes Greer.

\section{References}

1. Janardhan KS, Jensen H, Clayton NP, Herbert RA (2018) Immunohistochemistry in Investigative and Toxicologic Pathology. Toxicol Pathol 46: 488-510. [crossref]

2. Ghosh R, Gilda JE, Gomes AV (2014) The necessity of and strategies for improving confidence in the accuracy of western blots. Expert Rev Proteomics 11: 549-560. [crossref]

3. Aydin S (2015) A short history, principles, and types of ELISA, and our laboratory experience with peptide/protein analyses using ELISA. Peptides 72: 4-15. [crossref]

4. Ansotegui IJ, Melioli G, Canonica GW, Caraballo L, Villa E, et al. (2020) IgE allergy diagnostics and other relevant tests in allergy, a World Allergy Organization position paper. World Allergy Organ J 13: 100080. [crossref]

5. Kleinbeck ML, Hites MJ, Loker JL, Halliwell RE, Lee KW (1989) Enzyme-linked immunosorbent assay for measurement of allergen-specific IgE antibodies in canine serum. Am J Vet Res 50: 1831-1839. [crossref]

6. Lee KW, Blankenship KD, McCurry ZM, Esch RE, DeBoer DJ, et al. (2009) Performance characteristics of a monoclonal antibody cocktail-based ELISA for detection of allergen-specific IgE in dogs and comparison with a high affinity $\operatorname{IgE}$ receptor-based ELISA. Vet Dermatol 20: 157-164. [crossref]

7. Stedman K, Lee K, Hunter S, Rivoire R, McCall K, Wassom D (2001) Measurement of canine IgE using the alpha chain of the human high affinity IgE receptor. Vet Immunol Immunopathol 78: 349-355. [crossref]

8. Hensel P, Santoro D, Favrot C, Hill P, Griffin C (2015) Canine atopic dermatitis: detailed guidelines for diagnosis and allergen identification. BMC Vet Res 11: 196. [crossref]

9. Wang J, Godbold JH, Sampson HA (2008) Correlation of serum allergy (IgE) tests performed by different assay systems. J Allergy Clin Immunol 121: 1219-1224. [crossref]

10. Szeinbach, SL, Barnes, JH, Sullivan TJ, Williams PB (2001) Precision and accuracy of commercial laboratories' ability to classify positive and/or negative allergen specific IgE results. Ann Allergy Asthma Immunol 86: 1-9. [crossref]

11. Plant JD, Neradelik MB, Polissar NL, Fadok VA, Scott BA (2014) Agreement between allergen-specific IgE assays and ensuing immunotherapy recommendations from four commercial laboratories in the USA. Vet Dermatol 25: 15-16. [crossref]

12. DeBoer DJ, Hillier A (2001) The ACVD task force on canine atopic dermatitis (XVI): laboratory evaluation of dogs with atopic dermatitis with serum-based "allergy" tests. Veterinary Immunology and Immunopathology 81: 277-87. [crossref]

13. Codner EC, Lessard P (1993) Comparison of intradermal allergy test and enzymelinked immunosorbent assay in dogs with allergic skin disease. Journal of the American Veterinary Medical Association 202: 739-43. [crossref]

14. Hammerling R, De Weck AL (1998) Comparison of two diagnostic tests for canine atopy using monoclonal anti-IgE antibodies. Veterinary Dermatology 9: 191-199. [crossref]

15. Lee KW, Blankenship KD, McCurry ZM, McKinney B, Ruffner R, et al. (2012) Intra and inter-laboratory reproducibility of a monoclonal antibody cocktail based ELISA for detection of allergen specific IgE in dogs: proficiency monitoring of macELISA in six laboratories. Vet Immunol Immunopathol 148: 267-275. [crossref]

16. Enck K, Lee K, McKinney B, Lillard J, Globokar M, et al. (2020) Proficiency Monitoring of Allergen-Specific IgE macELISA - 2020. Integr J Vet Biosci 4: 1-7.

17. Mari A (2002) IgE to cross-reactive carbohydrate determinants: analysis of the distribution and appraisal of the in vivo and in vitro reactivity. Int Arch Allergy Immunol 129: 286-295. [crossref]
18. Holzweber F, Svehla E, Fellner W, Dalik T, Stubler S, et al. (2013) Inhibition of IgE binding to cross-reactive carbohydrate determinants enhances diagnostic selectivity. Allergy 68: 1269-1277. [crossref]

19. Levy BJ, DeBoer DJ (2018) A preliminary study of serum IgE against cross-reactive carbohydrate determinants (CCD) in client-owned atopic dogs. Vet Dermatol 29: 243-290. [crossref]

20. Lee KW, McKinney BH, Blankenship K, Morris, DO (2020) Detection and Inhibition of IgE for cross-reactive carbohydrate determinants evident in an enzyme-linked immunosorbent assay for detection of allergen-specific IgE in the sera of dogs and cats. Vet Dermatol 31: 439-e116. [crossref]

21. Piccione, ML, DeBoer DJ (2019) Serum IgE against cross-reactive carbohydrates determinants in healthy and atopic dogs. Vet Dermatol 30: 507-e153. [crossref]

22. Gedon, NKY, Boehm TMSA, Klinger CJ, Udraite L, Mueller RS (2019) Agreement of serum allergen test results with unblocked and blocked IgE against cross-reactive carbohydrate determinants (CCD) and intradermal test results in atopic dogs. Vet Dermatol 30: 243-e90.

23. Altmann F (2016) Coping with cross-reactive carbohydrate determinants in allergy diagnosis. Allergo J Int 25: 98-105. [crossref]

24. Foetisch K, Westphal S, Lauer I, Retzek M, Altmann F, et al. (2003) Biological activity of IgE specific for cross-reactive carbohydrate determinants. J Allergy Clin Immunol 111: 889-896. [crossref]

25. Jin C, Hantusch B, Hemmer W, Stadlmann J, Altmann F (2008) Affinity of IgE and IgC against cross-reactive carbohydrate determinants on plant and insect glycoproteins. J Allergy Clin Immunol 121: 185-190. [crossref]

26. Hemmer W, Altmann F, Holzweber F, Gruber C, Wantke F, et al. (2018) Immuno CAP cellulose displays cross-reactive carbohydrate determinant (CCD) epitopes and can cause false-positive test results in patients with high anti-CCD IgE antibody levels. J Allergy Clin Immunol 141: 372-381e3. [crossref]

\section{Citation:}

Lee KW, Esch RE (2021) Discovering the Pitfall of Using Horse Radish Peroxidase in Enzyme Linked Immunosorbent Assays for Detection of Pollen Specific IgE. Microbiol Immunol Pathol Volume 2(2): 1-8. 